Commun: Communication et organisation

Or

La communication, dimension oubliée de l'intelligence économique

\title{
Wargames et stratégies de communication
}

\section{Stéphane Goria}

\section{(2) OpenEdition}

Journals

Édition électronique

URL : http://journals.openedition.org/communicationorganisation/3903

DOI : 10.4000/communicationorganisation.3903

ISSN : $1775-3546$

Éditeur

Presses universitaires de Bordeaux

Édition imprimée

Date de publication : 1 décembre 2012

Pagination : 133-146

ISBN : 978-2-86781-772-4

ISSN : 1168-5549

Référence électronique

Stéphane Goria, «Wargames et stratégies de communication », Communication et organisation [En

ligne], 42 | 2012, mis en ligne le 01 décembre 2014, consulté le 19 avril 2019. URL : http://

journals.openedition.org/communicationorganisation/3903; DOI : 10.4000/

communicationorganisation.3903 


\title{
Wargames et stratégies de communication
}

\author{
Stéphane Goria'
}

L'année 2002 marque aux USA la naissance officielle des serious games avec les débuts de la Serious Game Initiative visant à promouvoir le développement de jeux vidéo pour des applications professionnelles (Amato, 2011). Ces jeux peuvent être considérés comme des applications informatiques "dont l'intention initiale est de combiner, avec cohérence, à la fois des aspects sérieux (serious) tels, de manière non exhaustive et non exclusive, l'enseignement, l'apprentissage, la communication, ou encore l'information, avec des ressorts ludiques issus du jeu vidéo (game) ... » (Alvarez et Djaouti, 2010, p. 17). Ce sont des outils relativement récents si on les considère uniquement du point de vue informatique, mais on peut aussi estimer que les premières pratiques de jeux sérieux datent des wargames utilisés par les militaires pour former leurs officiers et envisager de nouvelles stratégies ou tactiques (David et Sand, 2006, p. 51-52) (Marr, 2009, p. 29). Ils sont désormais aussi employés en intelligence économique sous le nom de business wargames (Miller, 2001) (Fuld, 2006, p. 69) (Besson et al., 2010, p. 71). Dans ce cadre, ils sont exploités pour simuler des situations de crises (Oriesek et Schwarz, 2008, p. 57-58), faire le bilan des forces et faiblesses d'une entreprise (Gilad, 2004, p. 95), préparer le lancement d'un produit (Herman et al., 2009, p. 112), tester des choix d'alliances stratégiques (Oriesek et Schwarz, 2008, p. 43) et anticiper l'évolution d'un marché (Gilad, 2009, p. 17). En fait, un wargame est une forme de serious game qui correspond à une tentative pour "recréer un affrontement réel ou imaginaire dans un espace et un temps définis » (Foley et Pierre-Pierre, 2007). En ce sens, le wargame peut être considéré comme un média de représentation (Frasca, 2003, p. 224). Il peut être utilisé comme un outil de visualisation d'informations stratégiques, d'exploration de choix possibles et de reconnaissance de faiblesses (David et Sand, 2006, p. 51). Appliqués à un cadre économique, les wargames comprennent de nombreux jeux de rôles associés à des cartes stratégiques réalisés dans un objectif d'anticipation d'une évolution de marché (Kurtz, 2003). Dans ce cas,

1 Stéphane Goria est Maître de conférences en Sciences de l'Information et de la Communication à l'Université de Lorraine, Laboratoire CREM ; Stephane.goria@univ-lorraine.fr 
au même titre qu'un jeu vidéo, un wargame est un média de simulation, car il incorpore « des règles de comportements, qui permettent à l'utilisateur de manipuler le modèle simulé "(Genvo, 2006, p. 216). En tant que tel, un wargame est jeu collectif qui possède une dimension communicationnelle très importante comme le partage de certaines valeurs et prémisses entre participants (Bateson et Ruesh, 1988, p. 243).

Si l'apport du wargame comme outil de réflexion et d'anticipation a déjà été abordé (Pelletier et Rodacachi, 2001) (Kurtz, 2003) (Schwarz, 2011), nous n'avons pas trouvé de publication spécifique aux stratégies de communication développées lors de séances de wargames ou faisant référence à des wargames. Ainsi, nous nous sommes demandé quels étaient les emplois potentiels et risques communicationnels des wargames. Nous avons parcouru la littérature des wargames et jeux sérieux similaires pour collecter de nombreuses informations se rapportant à cette question. Dans cet article, nous faisons part du résultat de cette revue de leur littérature en commençant par présenter les wargames à travers leur histoire, ce qui permettra de comprendre leur fonctionnement et leur apport en tant qu'outil de simulation pour l'aide au raisonnement stratégique. Puis, nous aborderons une série d'exemples de mise en ouvre des wargames pour explorer leurs différents risques et pratiques communicationnels que ce soit dans des contextes militaires, historiques ou économiques ${ }^{2}$.

\section{Histoire des wargames}

\section{Origines et premières exploitations}

Comme son nom l'indique, le wargame est à l'origine un jeu qui au fil des années est devenu un outil de simulation et de réflexion militaire. Son émergence est assez incertaine. On peut la faire remonter au $1^{\text {er }}$ millénaire avant J.-C., c'est-à-dire aux premières figurines de soldats (Ostermann, 1983, p. 9). Du point de vue des jeux de stratégie, sa lignée peut remonter au $\mathrm{VI}^{\mathrm{e}}$ siècle avant J.-C. avec l'utilisation en Chine du Wei Hai qui est l'ancêtre du jeu de Go et, à l'apparition en Inde (vers la même période) du Chaturanga qui est l'ancêtre des Échecs (Vitale, 1984, p. 9-10). Dès lors, trois branches distinctes des jeux de guerre vont évoluer conjointement : les jeux avec figurines, le jeu de Go et les Échecs.

Dans la ligne directe des jeux avec figurines, la mode des armées miniatures se répand dans les cours princières européennes au début du $\mathrm{XVII}{ }^{\mathrm{e}}$ siècle. Par exemple, G. Ostermann fait remarquer que le futur Louis XIII disposait

\footnotetext{
2 Nous considérons sur le même plan des wargames militaires, des wargames commerciaux et des business wargames. Cela peut paraître a priori surprenant, mais il semble que tous les auteurs d'articles et d'ouvrages sur les business wargames font référence à la littérature sur les wargames militaires et commerciaux. De plus, certains experts en business wargames sont aussi des concepteurs de wargames commerciaux, comme Mark Herman qui est cocréateur du jeu $S P Q R$.
} 
d'une armée "forte de plusieurs escadrons de cavalerie, d'infanterie et d'artillerie » qui " manœuvrait selon les mémorialistes de la cour, sur une "table percée" " (Ostermann, 1983, p. 10). Mais, c'est en 1773 qu'un cap important est franchi dans l'utilisation des figurines. Un écossais, J. Clerk, propose le premier wargame naval. Il est constitué de petits bâtonnets de bois figurants des navires de guerre et ses règles tiennent compte des effets du vent, des variations de puissance de feu des navires et de leur résistance aux dégâts. Il est considéré comme le premier véritable wargame avec figurines.

Concernant le Go, dès la dynastie des Hans (III' siècle après J.-C.), ce jeu est employé pour former l'esprit de l'élite de la société chinoise (Botermans, 2008, p. 300). Les Japonais l'adoptent vers le VIII siècle pour stimuler les capacités intellectuelles des jeunes officiers (Chamberland et Provost, 2008, p. 11). En 1603, le Shogun Iyeyasu étend cet objectif à toute la noblesse et au clergé en ouvrant la première académie de Go (Saucin, 1987).

Du côté des Échecs, au fil des années, certaines tentatives pour transformer ce jeu en outil de simulation de batailles font émerger en Prusse en 1644 les Échecs de guerre sous le nom de koenigspiel (jeu du roi). Ils sont plus tard perfectionnés par C.L. Helvig en 1780 qui dote le plateau de jeu de 1666 cases et de 120 pièces dans chaque camp à la place des 64 cases et 16 pièces pour chaque camp des Échecs traditionnels (Vitale, 1984, p. 10). Pour représenter des éléments de terrain (forêts, villages, cours d'eau, montagnes) certaines cases du plateau sont colorées et, pour faciliter le déroulement du jeu, un arbitre est introduit pour résoudre les affrontements entre pièces. Ensuite, un autre Prussien, G. Vinturinus, fait passer, en 1797, les Échecs de guerre d'une surface abstraite à une surface formée d'une carte topographique sur laquelle 3600 cases sont dessinées (ibid., p. 11). On peut estimer que le wargame sur carte vient d'être inventé.

\section{Passage du wargame militaire au wargame civil}

Le wargame sur plateau naît véritablement en Prusse en 1824, lorsque von Reißwitz le jeune propose l'adaptation d'un wargame avec figurines pour simuler avec précision une bataille sur un plateau cartographique. Le jeu est publié sous le titre : Instruction pour la représentation de manœuvres tactiques sous l'apparence d'un wargame ${ }^{3}$ (Perla, 1990, p. 25). Après quelques démonstrations et dizaines d'années, il sert à former les officiers prussiens et à les aider à préparer leurs batailles. Plus tard, les succès de l'armée prussienne face aux armées autrichienne et française dans la $2^{\mathrm{e}}$ moitié du XIX ${ }^{\mathrm{e}}$ siècle sont associés à l'emploi du wargame. Dès ce moment, son emploi est popularisé dans toutes les armées modernes. Le wargame prussien est étudié en Angleterre, aux États-Unis, en France, au Japon, en Russie, etc. (Patrick, 1977). Bientôt, deux variantes de ce wargame sont exploitées : le wargame libre et le wargame

3 Anleitung zur Darstelling militarische manover mit dem Apparat des Kriegßpiel. 
rigide. Le premier dit « libre » fait appel à un arbitre en lieu et place de règles de calculs pour résoudre les mouvements et engagements qui caractérisent le second « rigide » qui se passe d'arbitre et se fonde sur une quantité de calculs et de règles précises (ibid).

Suite à cet intérêt du wargame dans les armées du monde, celui-ci est très vite adapté pour la population civile. D'abord, aux USA, en 1880, C.A.L. Totten simplifie le wargame prussien en proposant un jeu destiné aux amateurs d'histoire militaire ${ }^{4}$ (Perla, 1990, p. 57). Du côté du wargame avec figurines (ou miniature wargame), une phase notable de son histoire a lieu en 1913 quand HG Wells publie les règles de Little Wars, un jeu destiné au grand public pour simuler des batailles (Patrick, 1977). Dans les années 1950, le wargame sur plateau cartographique passe à une phase commerciale démultipliant ainsi ses variantes (Palmer, 1977, p. 18). La première entreprise commerciale (Avalon Hill) dédiée à la commercialisation de wargames naît en 1958 aux USA (Perla, 1990, p. 115). D'autres suivent et un premier magazine (Strategy et Tactics) spécialisé dans les wargames paraît en 1968 (ibid. p. 129).

\section{Développement de nouvelles formes et applications}

Durant la Seconde Guerre mondiale, les wargames sont associés à des ordinateurs utilisés pour remplacer les tables de calculs des wargames rigides. Entre les années 1950 et 1970, les simulations de marchés commencent à se développer (Faria et Nulsen, 1996). Puis, au début des années 1970 le terme business wargame commence à être employé pour qualifier des simulations assez complexes assistées par ordinateur (Kalman et Rehnman, 1975). Parallèlement, au début des années 1970, G. Gygax concepteur de wargames avec figurines donne naissance au premier jeu de rôle (role playing game) commercial Donjons et Dragons (Gygax, 1987, p. 19-21). Ce jeu permet à quelques joueurs d'interpréter chacun un personnage lors d'une aventure contée par un maître du jeu qui remplace l'arbitre des wargames libres (Caïra, 2007, p. 45-46). Depuis, les jeux de rôle se sont largement répandus et une industrie particulière s'est créée tout autour d'eux (ibid. p. 28). Vers la fin des années 1970, certains business wargames sont conçus avec une dimension de jeu de rôle très importante (Oriesek et Schwarz, 2008, p. 19). Dans les années 1980, face à la montée en puissance des business wargames informatisés, certains auteurs dont B. Gilad défendent l'idée de business wargames « libres » fondés sur les atouts des jeux de rôles (Gilad, 2009, p. 30). Enfin, dans la seconde moitié des années 1990, les jeux de rôles commerciaux sur support informatique se transforment en jeux en ligne produisant ainsi la catégorie des $M M O R P G^{5}$ (Ichbian, 2011, p. 308) d'abord, puis dans les années 2000 celle des MMOFPS ${ }^{6}$ (Frostling-Henningsson, 2009).

4 STRATEGOS: a serie of american games of war based upon military principles.

5 Massively Multi-players Online Role-Playing Games.

6 Massively Multiplayer Online First-Person Shooter. 


\section{Les wargames comme outils de communication}

Les wargames sont à l'origine des jeux. Même lorsqu'ils sont appliqués à des activités sérieuses, leur nature ludique ne doit pas être pas complètement altérée (Alvarez et Djaouti, 2010, p. 26). C'est d'ailleurs en se fondant sur les jeux de rôle et les interactions entre participants suscitées par la dynamique du jeu que certaines connaissances sont présumées être créées lors d'une séance de wargame (Fuller et Loogma, 2009). Mais, de nombreux wargames ne se limitent pas simplement à leur aspect " jeu de rôle », ils utilisent aussi un support cartographique comme support visuel (Foley et Pierre-Pierre, 2007). En tant que tels, nous pouvons supposer qu'ils héritent des aspects communicationnels et des risques associés aux pratiques ludiques et cartographiques. En tant que jeu de rôle, un wargame "peut banaliser la réalité et attirer l'attention sur des détails présentant peu d'intérêt " (Chamberland et Provost, 2008, p. 87). Il peut aussi altérer la perception d'un des joueurs (Bowman, 2010, p. 138-139). Un joueur peut plaquer « ses représentations, voire ses préjugés, sur l'intrigue, sans ce soucier de la spécificité de l'univers du jeu » (Caïra, 2007, p. 250). De plus, si c'est un wargame exploitant une carte, il est dépendant des contraintes de son support. Or, une carte ne représente qu'une sélection d'informations et donc un ensemble de choix conceptuels (Paegelow, 2000, p. 130). La carte peut servir un objectif qui est caché par l'affichage des éléments qu'elle présente (Wood, 1992, p. 70). Les cartes peuvent même être employées comme des outils de propagande au service d'un certain pouvoir (ibid. p. 20). Comme média de représentation et de simulation, les wargames correspondent à des systèmes qui imposent des normes à leurs concepteurs et des choix conceptuels à leurs joueurs. Les uns comme les autres peuvent adapter ou détourner l'usage premier du jeu. Nous avons exploré la littérature sur les wargames et jeux de rôles sérieux pour y trouver quelques traces de ces pratiques et risques.

\section{Outil d'illustration ou d'affirmation identitaire}

Les wargames peuvent aider à donner du sens à certains propos. L'une de leurs premières utilisations fut l'assistance à la pédagogie. De ce point de vue, on peut considérer les mises en œuvre de wargames qui permettent à une personne de se servir du jeu comme support d'un discours. Ainsi, il est parfois difficile de savoir, lorsque quelqu'un fait la promotion d'un wargame, s'il le fait parce qu'il est convaincu de son efficacité ou bien s'il s'appuie sur le wargame pour faire valoir une différence vis-à-vis d'autres. Par exemple au XIX ${ }^{e}$ siècle, Helmut von Moltke a affirmé qu'il avait été convaincu très tôt de l'utilité du jeu de von Reißwitz (Patrick, 1977). Mais, comme les cadres de l'armée s'opposaient alors à son emploi, il est difficile de savoir si, en faisant la promotion du wargame tout au long de son ascension vers la tête de l'état major prussien, Moltke a défendu l'intérêt du wargame ou bien affirmé sa différence face aux vieux généraux. De même, en annonçant au 
monde l'apport du wargame pour les victoires prussiennes, on peut supposer qu'il a mis aussi en avant une certaine différence de pensée stratégique allemande (Perla, 1990, p. 30). De manière similaire, Mao Zedong annonça dans plusieurs de ses discours que sa pensée stratégique était fondée sur le jeu de Go (Boorman, 1972, p. 14-15). De cette manière, Mao Zedong a pu se servir du Go comme métaphore pour illustrer sa pensée auprès de son public. Toutefois, il n'en demeure pas moins que, de fait, il s'est servi du Go pour affirmer une différence de pensée stratégique chinoise vis-à-vis d'une autre occidentale (Boorman, 1972, p. 15).

On peut aussi se servir de ce jeu comme d'un outil d'affirmation ou bien de revendication identitaire. Les travaux de S.A. Boorman (1972) vont dans ce sens. Il a utilisé le jeu de Go et ses principes en se fondant sur l'hypothèse que la stratégie militaire de Mao Zedong était fondée sur ce jeu (voir ci-dessus). Il a transformé certaines cartes de la Chine en plateau de Go en associant les lignes de communication aux lignes du plateau de Go. Il a montré ainsi que la stratégie de Mao Zedong pouvait s'interpréter comme une stratégie de joueur de Go (figure 1). J. Saucin (1987) utilisa lui aussi le Go dans une optique pédagogique pour démontrer que les stratégies des gouvernements japonais depuis 1867 sont implicitement des stratégies de Go (voir chapitre suivant).
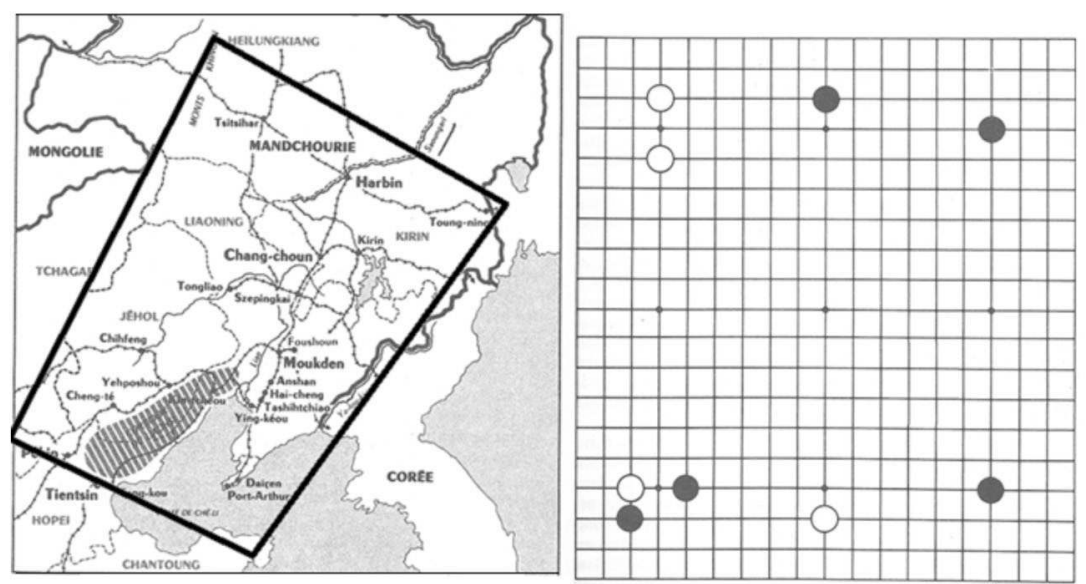

Figure 1 - Positions au début de la campagne de Mandchourie des communistes en noirs et des nationalistes en blancs (d'après Boorman, 1972, p. 134-135).

Les Échecs ont aussi été défendus pour leur qualité pédagogique dans le cadre de l'explication d'une bataille (Palhares, 2007). Ils ont été utilisés par Ansur (1907) pour expliquer la bataille de Chryssus ${ }^{7}$ entre les armées omeyyade

7 Ou bataille du Guadalete. 
et wisigothe. Son système de transposition est fondé sur la chronologie de la bataille, la présence d'un chef d'armée dans chacun des deux camps et un rapport de mouvement entre pièces d'Échecs et troupes réelles ${ }^{8}$. Dans le même ordre d'idées, A. Calhamer, créateur du jeu de plateau Diplomacy, s'est servi de son jeu pour illustrer les enjeux diplomatiques et géopolitiques entre puissances européennes tout au long du XX $\mathrm{X}^{\mathrm{e}}$ siècle (Calhamer, 1999). En fait, la très grande majorité des wargames commerciaux peuvent servir de supports pédagogiques pour expliquer un conflit en mettant en évidence «les éléments prévalant dans la prise de décision des chefs militaires de chaque camp » (Liardet, 1997, p. 327).

Lors d'une séance de wargame, une dynamique de jeu est créée par différents éléments qui sont là pour être manipulés (Dunnigan, 2000, p. 114). Un joueur peut transformer le jeu selon l'idée qu'il souhaite exprimer en fonction de sa compréhension de la partie en cours. Y. Yasuda a montré que l'utilisation du Go permettait d'établir un dialogue avec des enfants (Yasuda, 2003, p. 24-25), des personnes handicapées (ibid. p. 35) ou des personnes âgées (ibid. p. 46-47). Cependant, l'utilisation du jeu peut quelquefois être détournée par certains joueurs. Au Sénégal, dans le cadre de la mise en œuvre de jeux de rôle en vue de l'amélioration de la gestion de systèmes d'irrigation, W. Daré $(2005$, p. 253) a noté que lors des séances de jeux, certains joueurs ne se sont pas consacrés à l'objectif du groupe. Ils ont simplement tenté de s'affirmer individuellement comme de "bons agriculteurs ». Dans un registre très similaire, des cartes et des jeux de rôles étaient employés pour aider des groupes de personnes à développer un projet commun de demande foncière. L. Boutinot et al. (2008) ont remarqué que certains joueurs se sont servis des séances comme d'une seconde chance pour défendre certaines revendications qui n'avaient pas été prises en compte dans un contexte non ludique.

Le support du jeu lui-même peut servir d'outil de revendication ou de propagande. Un premier cas de ce genre est peut-être le wargame de Venturini. Nous rappelons qu'en 1797, il crée les Échecs de guerre sur carte. Or, la carte choisie pour les démonstrations de ce jeu prussien est la frontière franco-belge (Palmer, 1977, p. 16). Il nous semble peu probable que ce choix ne soit pas lié au contexte géopolitique de l'époque. Bien des années plus tard, le jeu de guerre sur informatique peut aussi devenir un instrument de propagande. G. Frasca (2003, p. 232) relève que quelques modifications simples du MMOFPS Quake permettent d'obtenir un jeu d'affrontement entre soldats israéliens et palestiniens.

\section{Outil de démonstration objectif ou prisonnier d'un dogme}

Le wargame peut servir d'outil de démonstration d'une thèse. L'Amiral Nimitz l'utilisa ainsi en 1923 pour démontrer l'importance des porte-avions

8 Les pions correspondent aux troupes à pied et toutes les autres pièces sauf le roi correspondent à de la cavalerie. 
dans les prochains conflits en mers (Liardet, 1997, p. 33). Mais pour ce faire, un jeu a dû être conçu pour simuler les capacités supposées des porte-avions. Le wargame lui-même à travers ses mécanismes était, en quelque sorte, vecteur de la thèse de Nimitz. Nous pouvons retrouver ce cas de figure, notamment, dans le cadre de wargames historiques. Deux jeux peuvent représenter la même bataille à la même échelle sur une carte topographique pavée en hexagones, tout en ayant des mécanismes des jeux bien différents.

Les systèmes de règles des jeux Battles of Ancient World (Decision Games) et $S P Q R(G M T)$ proposent de traiter la bataille de Cannes opposant l'armée d'Hannibal aux légions romaines. Mais, si on regarde la disposition respective des troupes au début de la bataille pour ces deux jeux, nous nous apercevons du premier coup d'œil que certains choix positionnels et numériques ont été effectués (figure 2).
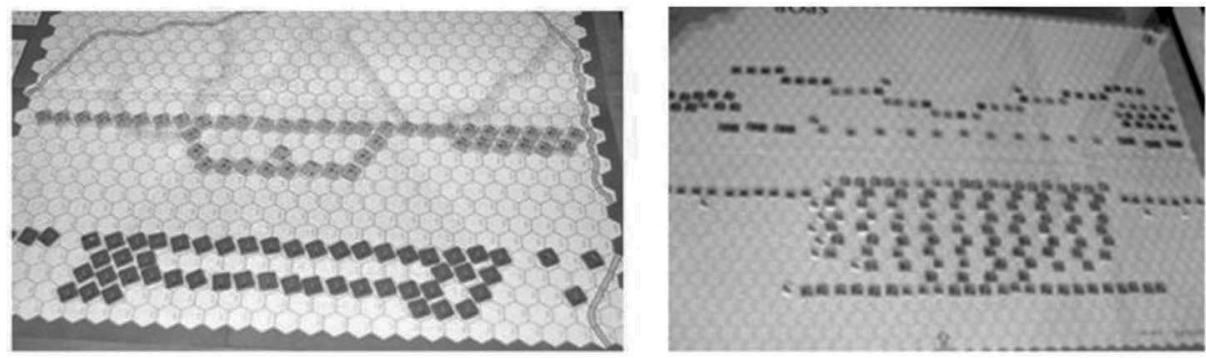

Figure 2 - Représentation de la bataille de Cannes (armée romaine en bas et carthaginoise en haut) avec Four Battles of Ancient World (gauche) et SPQR (droite)

Cette différence de traitement est encore plus flagrante si on compare les informations affichées sur les pions (figure 3) et leur rapport aux règles. Les pions de Battles of Ancient World portent deux informations numériques contre trois pour ceux de $S P Q R$. Le troisième chiffre des pions de $S P Q R$ qui n'apparaît pas dans Battles of Ancient World est lié au moral des troupes. D'après les règles, les jets de moral sont très importants à $S P Q R$, car dès qu'une troupe en affronte une autre, elle doit en effectuer. Si un jet de dés traduit une baisse de morale pour une troupe, celle-ci est inactive ou part en déroute (Herman et Berg, 1992, p. 24-25). Le résultat de la bataille à $S P Q R$ dépend donc beaucoup du moral des troupes. Or, ces tests de moral sont l'interprétation, sous la forme de mécanismes
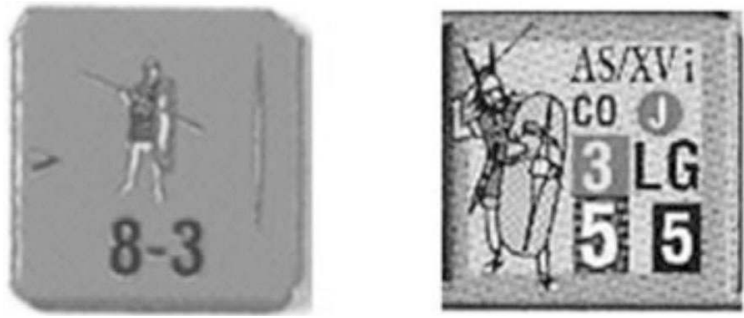

Figure 3 - Aperçu de pions dans Four Battles of Ancient World (gauche) et SPQR (droite) 
de jeu, des travaux sur l'armée romaine de H. Delbruck (1990) lui-même très influencé par les théories de C. Ardant du Picq (2004) $)^{9}$ sur l'importance de la psychologie sur le comportement des individus lors d'un combat.Nous pouvons considérer de même les choix de J. Saucin (1987) pour montrer que, depuis plus d'un siècle, le développement économique du Japon correspond à des choix stratégiques de joueurs de Go. La thèse qu'il défend est que les managers japonais font de manière inconsciente des choix stratégiques que l'on retrouve dans le jeu de Go. Pour défendre ce point de vue, il propose de trier par proximité et importance économique différents secteurs d'activités du marché japonais (alimentation, textile, éducation, sidérurgie, construction navale, etc.) pour les associer à des secteurs du plateau de Go. Les coins du plateau représentent les secteurs les plus primaires et le centre du plateau les secteurs les plus évolués comme la construction navale. Il associe les pièces noires aux coups joués par les nations occidentales sur l'ensemble du marché japonais et les pierres blanches aux les coups joués par le gouvernement japonais.

Le biais induit par les prémisses de cette présentation d'information concerne la latitude dont dispose J. Saucin à l'intérieur de chaque zone pour poser les pierres selon une stratégie de Go. Il augmente de fait la perception de certains ensembles de pierres comme des encerclements. Par exemple, sa représentation (figure 4) permet de voir, dans l'optique d'une partie de Go, le développement du Japon face aux nations occidentales entre 1867 et 1937.

Les choix conceptuels lors de la création d'un jeu sont donc très importants et loin d'être neutres. L'histoire nous a laissé de nombreux rapports de séances de wargames qui avaient été victimes de défauts conceptuels ou mécaniques. Les séances qui en découlent ne sont pas objectives, mais représentent ou défendent un certain dogme. Ainsi, quelques parties de wargames sont restées célèbres dans l'histoire. Parmi celles-ci, le contexte d'élaboration du plan Schlieffen est assez exemplaire. L'armée allemande très habituée à utiliser le wargame comme outil d'aide $\mathrm{au}$ raisonnement fut prise au piège d'idées préconçues. Au début du $\mathrm{XX}^{\mathrm{e}}$ siècle, de nombreuses simulations d'attaques de la France furent réalisées. Ces séances tenaient compte du risque d'une offensive russe sur le front est,

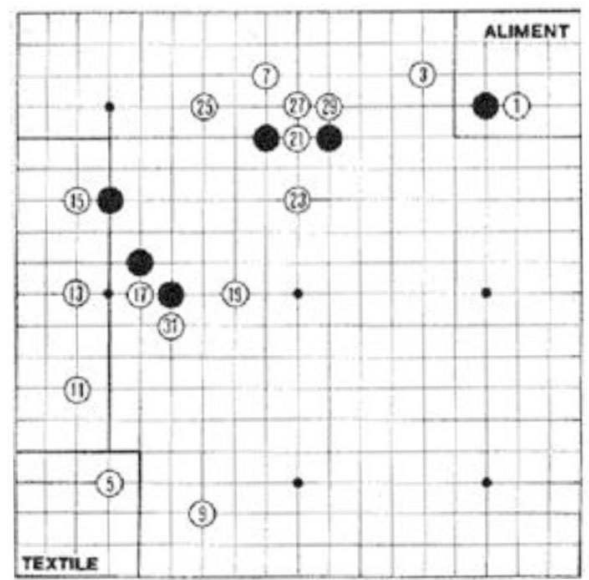

Figure 4 - Développement économique du Japon entre 1867 et 1937 d'après (Saucin, 1987)

9 D’après F. BEY, «SPQR : La légion romaine est-elle invincible ? », Vieille Garde, n6, 1996, 12 p. 
en réaction de l'attaque du front français (ouest) conformément à l'alliance liant la France et la Russie. Toutefois, l'état major allemand ignora jusqu'en 1914 toute possibilité d'intervention de l'Angleterre au côté de la France (Vitale, 1984, p. 13). La bataille de Midway est un autre exemple typique qui nous est resté d'une mauvaise exploitation du wargame. Début mai 1942, la marine japonaise consacra quatre jours à des séances de wargames pour préparer l'invasion de l'île de Midway. D'abord, le nombre de séances prévues pour les parties fut trop faible pour envisager suffisamment de cas de figure dangereux pour la marine japonaise, comme la présence dès le début de partie de porte-avions américains au nord-est des positions japonaises (Perla, 1990, p. 46-47), ce qui se produisit lors de la bataille réelle. Ensuite, l'aviation japonaise, dans tous les cas, était supposée plus forte que sa rivale américaine tandis que les porte-avions japonais coulaient moins facilement que leurs homologues américains (ibid. p. 46-47).

\section{Conclusion}

Nous avons abordé dans cet article les wargames sous l'angle communicationnel. Cela nous a permis de mettre en évidence certains de leurs aspects en dehors de leurs seuls apports en tant qu'outil de réflexion historique, de prospective ou de simulation. Nous avons vu que les wargames sont exploitables pour promouvoir une différence de pensée ou permettre à des personnes de s'exprimer sur une question stratégique. De même, ils peuvent servir de support à une communication stratégique en associant des images symboliques d'une stratégie de jeu à certaines idées abstraites. Fondés sur le partage et la diffusion de connaissances entre personnes et l'étude de choix possibles, les wargames peuvent être aussi considérés comme des outils de création et capitalisation de connaissances. Mal utilisés, ils peuvent servir d'outil de propagande ou être prisonniers d'un dogme. Mieux utilisés, ils peuvent être employés pour faire la promotion d'une idée ou bien fédérer des personnes autour d'un projet. La gestion de la communication associée à une partie de wargame est donc plus complexe à réaliser qu'il n'y paraît a priori. Dans des cadres d'intelligence économique, l'enjeu d'un bon management de la communication interpersonnelle ou d'une mise en forme adéquate, claire et non ambiguë d'informations lors de séances de wargames est donc très important pour le succès de cette pratique. Le risque d'une simulation fondée sur un dogme implicite est d'ailleurs peut-être le plus grand danger que font encourir ces types de serious games à leurs utilisateurs. 


\section{BIBLIOGRAPHIE}

ALLEN T., "The evolution of wargaming: From chessboard to marine doom ", in CORNELL T., THOMAS A. (sous la direction de), War and Games, San Marino, 2002, p. 231-251.

ALVAREZ J., DJAOUTI D., Introduction au Serious Game, Questions Théoriques, Quercy, 2010, 227 p.

AMATO E. A., «Les utilités du jeu vidéo sérieux : finalités, discours et mises en corrélation ", La Revue canadienne de l'Apprentissage et de la Technologie, 2011, volume 37, n², p. 1-19.

ANSUR A., O Jogo Real - Apontamentos diversos para a tentativa de um tratadinbo de xadrez, Lisboa, Tupographia do Commércio, de Leiria, Abilio Saraiva, 1907, 334 p.

ARDANT du PICQC., Études sur le combat : Combat antique et Combat moderne, Economica, Paris, 254 p.

BATESON G., RUESCH J., Communication et société, Le Seuil, Paris, 1988, 346 p.

BESSON B., DESCHAMPS C., ANDRIAMIAMINA H. et al, Méthodes d'analyses appliquées à l'intelligence économique, ICOMTEC, Poitiers, 2010, 127 p.

BEY F., "SPQR : La légion romaine est-elle invincible ? ", Vieille Garde, n6, 1996, p. $3-15$.

BOORMAN S. A., Gô et Mao : pour une interprétation de la stratégie maoïste en termes de gô, Éditions du Seuil, Paris, 1972, 216 p.

BOTERMANS J., The Book of Games: strategy, tactics et history, Sterling Publishing, 2008, 736 p.

BOUTINOT L., VIAU A., LECLERC G., «Questions sur la neutralité des outils de type jeux de rôle et cartographie participative dans une expérience de gouvernance foncière au Sénégal », Norois, 2008, volume 209, n4, p. 1-17.

BOWMAN S. L., The Functions of Role-Playing Games: How the Participants Create community, Solve Problems and Explore Identity, McFarland et Company Inc Publishers, Jefferson, 2010, 208 p.

CAÏRA O., Jeux de rôle : Les forges de la fiction, CNRS Éditions, Paris, 2007, 311 p.

CALHAMER A., Calhamer on Diplomacy: The Boardgame Diplomacy and Diplomatic History, Books Library, 1999, 223 p.

CHAMBERLAND G. PROVOST G., Jeu, simulation et jeu de rôle, Presses de l’Université du Québec, Québec, 2008, 178 p.

CHAMBERLAND G., LAVOIE L., MARQUIS D., 20 formules pédagogiques, Saint-Foy : Presses de l'Université du Québec, Québec, 1995, 196 p.

CRAWFORD C., The Art of Computer Game Design, Osborne - McGraw-Hill, 1982, $120 \mathrm{p}$.

DAVID M. SANDE C., Serious Games: Games that educate, train and inform, Thomson course Technology, Boston, 2006, 352 p.

DARE W., Comportements des acteurs dans le jeu et dans la réalité : indépendance ou correspondance? Analyse sociologique de l'utilisation de jeux de rôles en aide à la concertation, 
Thèse, Ecole Nationale du Génie Rural, des Eaux et Forêts (ENGREF) Centre de Paris, 2005, 397 p.

DELBRUCK H., Warfare in Antiquity: History of the Art of War, Vol.1, University of Nebraska Press, 1990, 607 p.

DUNNIGAN J., Wargames Handbook, Third Edition: How to Play and Design Commercial and Professional Wargames, iUniverse.com, 2000, 418 p.

FARIA T. AJ, NULSEN R., « Business simulation Games: current usage levels: A ten year update ", Developments In Business Simulation et Experiential Exercises, 23, 1996, 7 p.

FRASCA G., Videogames of oppressed: videogames as a mean of critical thinking and debate, Master Thesis, Georgia Institute of technology, 2001, 318 p.

FOLEY C., PIERRE-PIERRE A., « Qu'est-ce que le wargame? Une introduction rapide ", Vae Victis, Revue du Jeu d'Histoire Tactique et Stratégique, HS n6, 2007, p. 4-11.

FULD L., The Secret Language of Competitive Intelligence: How to See Through and Stay Ahead of Business Disruptions, Distortions, Rumors, and Smoke Screens, Crown Business, 2006, $320 \mathrm{p}$.

FROSTLING-HENNINGSSON M., "First-Person Shooter Games as a Way of Connecting to People: "Brothers in Blood" ", CyberPsychology E Behavior, 12, 2009, n5, p. 557-562.

FULLER T., LOOGMA K., " Constructing Futures; a social constructionist perspective on foresight methodology ", Futures; Journal of Policy, Planning and Futures Studies, 41, 2009, n², 17 p.

GILAD B., Business War Games: How large, small, and new companies can vastly improve their strategies and outmaneuver the competition, Career press, Franklin Lakes, 2009, $224 \mathrm{p}$.

GILAD B., Early Warning: Using Competitive Intelligence to Anticipate Markets Shifts, Control Risk, and Creat Poweful Strategies, Amacom Books, New York, 2004, 268 p.

GENVO S., Le game design de jeux vidéo : approche communicationnelle et interculturelle, Thèse en Sciences de l'information et de la Communication, Université Paul Verlaine, 2006, 406 p.

HERMAN M., BERG R., SPQR : Règles du jeu, Oriflam, 1992, 52 p.

HERMAN M., FROST M. et KURZ R., Wargaming for leaders: Strategic decision making from the battlefield to the boardroom, McGraw Hill, Chicago, 2009, 275 p.

ICHBIAN D., La Saga des jeux vidéo, Éditions Pix'n Love, 2011, 521 p.

KALMAN C., RHENMAN E., "The Role of Management Games in Education and Research ", in GREENBLAT C. et DUKE R. (sous la direction de), Gaming-Simulation: Rationale, Design and Application, Sage Publication, New York, 1975, p. 233-269.

KURTZ J., " Business wargaming: simulations guide crucial strategy decisions ", Strategy et Leadership, 2003, 31, n 6, 2003, p. 12-21.

LIARDET J.-P., Les wargames commerciaux américains des années soixante à nos jours, entre histoire militaire et simulation, une contribution à l'étude de la décision, Tome 1, Septentrion Presses Universitaires, 1997, 386 p.

MARR A. C., Serious Games für die Informations-und Wissensvermittlung, Bibliotheken auf neuen Wegen, Stuttgart, 2009, 165 p. 
MILLER A., "Competitive intelligence - an overview ", Competitive intelligence Magazine, 2001, 12, $\mathrm{n}^{\circ}$ 4, $14 \mathrm{p}$.

ORIESEK D., SCHWARZ J. O., Business Wargaming: Securing Corporate Value, Gower, Aldershot, 2008, 136 p.

OSTERMANN G., Les jeux de stratégie et de tactique historiques : historique, typologie, perspectives, Thèse de doctorat, Centre militaire et d'études de la défense nationale, Université Paul-Valéry Montpellier III, 1983, 157 p.

PALHARES P., "The use of the game of chess to represent famous battle ", Board Game Studies, Colloquium XI, Lisbon, 2007, p. 146-154.

PALMER N., The Comprehensive Guide to Board Wargaming, Arthur Baker Ltd, london, 1977, 222 p.

PATRICK S., "The History of Wargaming ", in Wargame Design: The History, Production and Use of Conflict Simulation Games, Simulations Publications Incorporated, 1977, p. 1-40.

PELLETIER E., RODOCANACHI P., «Un outil d'anticipation des risques : le wargame ", L'expansion Management Review, 2001, volume décembre, p. 106-110.

PERLA P., The art of Wargaming, Naval Institute Press, Annapolis, 1990, 366 p.

RAVOIRE J.-P., " Pour aller plus loin : quels jeux pour quels joueurs ? ", Vae Victis, Revue du Jeu d'Histoire Tactique et Stratégique, HS n6, 2007, p. 58-69.

SALEN K., ZIMMERMAN E., Rules of Play: Game Design Fundamentals, MIT Press, 2004, 688 p.

SAUCIN J., « Jeu de Go et management japonais », L'aperçu Trimestriel Économique, volume avril, 1987, p. 1-26.

SCHWARZ J. O., "Ex ante strategy evaluation: the case for business wargaming ", Business Strategy Series, 2011, volume 12, n³, p. 122 - 135.

VITALE D., Jeux de simulation : wargames, M.A. Éditions, Evreux, 1984, 256 p.

WOOD D., The Power of Maps, The Gilford Press, New York, 1992, 248 p.

Résumé : Les wargames sont des jeux utilisés notamment par les professionnels pour simuler et préparer des stratégies d'entreprise. Leur usage remonte au moins au XVIII siècle quand les militaires ont adapté un jeu de guerre pour former des officiers au commandement. Désormais, ils sont employés en intelligence économique sous le nom de business wargames. Ils correspondent à une mise en œuvre ciblée et coordonnée de jeux de rôles et de représentations cartographiques pour répondre à un objectif stratégique particulier. Si leur utilité en tant qu'outil d'aide au raisonnement ou à l'anticipation stratégique a déjà été étudiée, nous avons constaté que leurs usages et pratiques communicationnels n'étaient abordés que de façon éparse. Nous avons souhaité rassembler dans cet article diverses informations et expériences se rapportant à la question des potentiels et risques communicationnels des wargames que cela soit dans des contextes militaires, économiques ou historiques.

Mots-clés : Wargame, War Game, jeu sérieux, jeu de rôle, communication interpersonnelle, stratégie de communication, visualisation d'information. 
Abstract : Wargames are a kind of games used notably by professionals to simulate and prepare business strategies. Their use goes back to the eighteenth century at least, when the armies have adapted a war game to train officers to take command. Nowadays, they are used in economic intelligence with the name of business wargames. They are a particular and coordinated adaptation of a role playing games and maps to solve a strategic problem. If their usefulness as a tool to help for reasoning or the strategic anticipation has been studied, we observed that works about their uses and communicational practices were strewn. Thus, we have wanted to gather in this paper a set of information and experiences related to the issue of potentialities and communicational risks of wargames whether in military contexts, economic or historical.

Keywords : Wargame, War Game, Serious Game, Role Playing Game, Interpersonal communication, Communication strategy, Information visualization. 